\title{
Ambiguity, Pessimism, and Religious Choice
}

\author{
Tigran Melkonyan \\ Professor of Economics \\ University of Nevada, Reno \\ tmelkonyan@cabnr.unr.edu
}

and

\author{
Mark Pingle \\ Professor of Economics \\ University of Nevada, Reno \\ pingle@unr.edu
}

\begin{abstract}
Using a relatively mild restriction on the beliefs of the $\alpha$-MMEUpreference functional, in which the decision maker's degree of ambiguity and degree of pessimism are each parameterized, we present a rather general theory of religious choice in the decision theory tradition, one that can resolve dilemmas, address the many Gods objection, and address the inherent ambiguity. Using comparative static analysis, we are able to show how changes in either the degree of ambiguity or the degree of pessimism can lead a decision maker to "convert" from one religion to another. We illustrate the theory of religious choice using an example where the decision maker perceives three possible religious alternatives.
\end{abstract}


“...I know ...I must soon die, but what I know least is the very death I cannot escape." --- Blaise Pascal (1670 [1958], fragment 194)

\section{Introduction}

Suffering from ill health most of his adult life, Blaise Pascal experienced what he said knew least on August 16,1662 at age 39. Exiting his short life, he left behind a legacy of significant contributions to math, science, and religious philosophy. Today, a unit of pressure, a computer programming language, and a triangle of numbers bear his name. Pascal shifted away from studying math and science, after a moving personal experience in 1654 led to his "second conversion. " He was working on a comprehensive apology for the Christian faith, but died before completing it. Pascal's Pensées (Pascal, 1670 [1958]), a collection of Pascal's thoughts on religion and philosophy, was published posthumously. Among these thoughts, one finds an innovative application of logic to religious choice, thinking that has been labeled "the advent of decision theory" (Jorden, 1994a, p.3).

It may not be incidental that thinking about life after death played a role in the development of decision theory. What happens at the moment of death may be the quintessential example of uncertainty. This uncertainty, like most, tends to make people anxious. Adam Smith (1759 [2000], Part I, paragraph I.I.13) called the "dread of death" the "great poison to human happiness.”

Religion can ameliorate the dread associated with death. Religious precepts typically instruct people about life and the afterlife, and some of this teaching may reduce the uncertainty

\footnotetext{
${ }^{1}$ Pascal's "first conversion" was a 1646 commitment to Jansenism, a relatively Puritanical and relatively Augustinian practice of Roman Catholicism that conformed to the writings of Cornelius Jansen. The "second conversion" is associated with an event reportedly occurring on November 23, 1654. Pascal and some friends were riding in a carriage when the team of horses pulling it plunged off a bridge. Because the reins broke, the carriage did not follow, but was left half on and half off the bridge. Pascal apparently fainted out of a fear of the nearness of death, and was unconscious for some time. Later, while recovering, Pascal had what he described as an intense religious vision. The notes he immediately took down to remind himself of the vision, known as the Memorial, were inadvertently found by a servant after his death, sewn into Pascal's coat.
} 
a person associates with death. Pascal's innovation was to recognize that, even if religious teaching does not eliminate uncertainty, a rational faith decision can still be made by considering the anticipated payoffs in various possible states.

In its most simple form, Pascal's "wager" can be interpreted as a choice between theism and atheism. In Pascal's words:

God is or He is not. But to which side will we incline? ... What will you wager? ... You must wager. It is not optional ... Let us weigh the gain and the loss in wagering that God is. ... If you win, you win everything, if you lose you lose nothing. Do not hesitate then; wager that he does exist.

--- Pascal（1670 [1958], fragment 233)

In these words, we see fundamental elements of modern decision theory. The choice set is parsed into a set of mutually exclusive alternatives: "Wagering that God is" and "wagering that God is not." Uncertainty is recognized by positing mutually exclusive potential states: "God is" and "God is not." A payoff is associated with each alternative-state combination. In this wager, the payoffs are not explicit, but Pascal implicitly assumes one decision alternative "weakly dominates" the other. Thus, in this pioneering decision theory problem, the weak dominance concept was applied to offer one explanation for why it is rational for people to choose theism over atheism.

Of course, the assumptions Pascal imbedded in his original wager can be modified, so that the decision theory must be extended in order to explain choice. For example, McClennan (1994, p.118) remarks, "Many will simply reject the claim that, if God does not exist, you have lost nothing by betting on God." If payoffs are altered to suit this statement, the wager may 
become a dilemma the best choice depends upon the state that arises. Because weak dominance does not hold in this case, it cannot be used to identify the rational choice. To predict the choice, decision theory must be extended to include a decision criterion that will resolve the dilemma.

The "many Gods objection" is another significant critique of Pascal's wager. This is the recognition that, for many people, the set of religious alternatives contains more than two elements. ${ }^{2}$ To address the many Gods objection, a decision theory must be applied that can accommodate more than two decision alternatives.

Subjective Expected Utility (SEU) theory (Savage, 1954) can resolve dilemmas and accommodate many alternatives, so we might try to use it to construct a more general theory of religious choice. However, the inability to collect objective religious information makes the applicability of SEU theory questionable (Montgomery, 1996). Death, according to Shakespeare (Hamlet, Act 3), is "the undiscovered country from whose bourn no traveler returns." What happens after death may be so uncertain that a decision maker cannot construct a unique subjective probability distribution over alternative religious states, nor rationally update beliefs.

The inability to construct a single probability distribution over the states is what distinguishes "ambiguity" from "risk." The distinction between risk and ambiguity dates to Knight (1921[1971]) and Keynes (1921). However, models of choice under ambiguity have only recently been developed, largely to explain Ellsberg (1961) behavior, the finding that people respond to ambiguity in ways that contradict SEU theory. Because such contradictions

\footnotetext{
${ }^{2}$ See Jordan (1994b) for a discussion of the many Gods objection. Hacking (1994) and Morris (1994) suggest Pascal did not intend for us to partition the set of choices into theism and atheism. Rather, it is more accurate to say Pascal's intent was to make the partition "pursuing God" with actions that will probably lead one to believe versus "not bothering about such things" (Hacking, 1994, p.25). Similarly, Morris (1994, p56) contends Pascal's intent was to use his wager to put the unbeliever on the path to belief in the Christian faith which Pascal had adopted. However, the many Gods objection remains as long as one admits the possibility that all do not find the same religion in the search.
} 
have repeatedly and routinely been found in the experimental and empirical literature, ${ }^{3}$ we have reason to expect that the SEU model may fail to explain religious choice and that a model of ambiguity may perform better. The most popular models capable of explaining Ellsberg-type behavior are Schmeidler's (1989) Choquet expected utility (CEU) model and Gilboa and Schmeidler's (1989) maximin expected utility (MMEU) model.

The $\alpha$ - maximin expected-utility ( $\alpha$-MMEU) model (see, e.g., Cohen, 1992, Jaffray and Philippe, 1997, Ghirardato, Klibanoff and Marinacci, 1998, Ghirardato, Maccheroni and Marinacci, 2004, Siniscalchi, 2006, Ludwig and Zimper, 2006, Olszewski, 2008, Eichberger, Grant and Kelsey, 2008) utilized in this paper is a generalization of the MMEU model. The general nature of $\alpha$-MMEU model makes it especially suitable for modeling religious choice. Beliefs are represented by a set of probability distributions. The model reduces to the Savage (1954) SEU model when the set of distributions is a singleton, capturing a decision maker who happens to be able to construct a probability distribution over different religious states. At the opposite extreme, it reduces to the Arrow and Hurwicz (1972) model of total ambiguity when the set of distributions becomes the probability simplex, capturing a decision maker who regards religious choice as being totally ambiguous. Between these two extremes, the size of the set of probability distributions is a measure of the degree of ambiguity, or a measure of what Gajdos et al (2004) refer to as the degree of information imprecision.

Religious alternatives in the $\alpha$-MMEU model are evaluated using a convex combination of minimum and maximum expected utilities. The relative weight placed on the probability distribution that minimizes expected utility can be interpreted as a measure of ambiguity aversion or, following Arrow and Hurwicz (1972), a measure of pessimism. A totally ambiguity

\footnotetext{
${ }^{3}$ Camerer and Weber (1992) and Camerer (1999) provide accessible introductions to the empirical literature on the Ellsberg Paradox.
} 
averse decision-maker, or a total pessimist, places all of the weight on the probability distribution that minimizes expected utility. Alternatively, a totally ambiguity tolerant decision-maker, or a total optimist, places all of the weight on the probability distribution that maximizes expected utility. Thus, the $\alpha$ - MMEU model has the advantage of having maximin expected utility and maximax expected utility as special cases.

Our contribution in this paper is twofold. First, we derive a number of comparative static results for an $\alpha$-MMEUdecision maker whose beliefs are given by the core of a simple capacity. Specifically, we examine how choice is affected as the degrees of ambiguity and pessimism interact in the $\alpha-\mathrm{MMEU}$ model. Second, we use this preference functional to present a rather general theory of religious choice in the decision theory tradition, one that can resolve dilemmas, address the many Gods objection, and address the ambiguity inherent in the choice. Using straightforward comparative static analysis, we are able to show how changes in either the degree of ambiguity or the degree of pessimism can lead a decision maker to "convert" from one religion to another. We illustrate our results using an example where the decision maker perceives three possible religious alternatives.

\section{Parameterizing Ambiguity in an $\alpha$-MMEUModel}

Assume a decision-maker DM must choose an action $x$ from $N$ mutually exclusive actions $X=\{1,2, \ldots, N\}$. DM perceives the payoff provided by action $x$ depends upon which state $\theta$ arises from among $N$ mutually exclusive states $\Theta=\{1,2, \ldots, N\}$. In particular, DM perceives the payoff $u_{\theta}^{x}$ will be received when action $x$ is chosen and state $\theta$ occurs. 
To apply this framework as a model of religious choice, let the action be the choice to adopt religion $x \in X$. Assume DM is able to arrange his or her conceptions of different religions into $N$ mutually exclusive alternatives. Also, assume DM believes one and only one religion is true. Uncertain as to which is true, DM perceives $N$ possible states, where $\theta \in \Theta$ is the state "religion $\theta$ is true." DM perceives the payoff $u_{\theta}^{x}$ from adopting religion $x$ under state $\theta$. To have a specific example, suppose DM perceives three religious alternatives with the payoffs presented in Table 1.

Table 1: Illustrative Religious Decision Matrix for DM

\begin{tabular}{|l|c|c|c|}
\hline & Religion a True & Religion b True & Religion c True \\
\hline Religion a & 10 & -20 & -20 \\
\hline Religion b & -10 & 20 & -10 \\
\hline Religion c & -20 & -10 & 30 \\
\hline
\end{tabular}

DM's beliefs may be ambiguous, meaning the likelihoods of the different states in $\Theta$ may not be known with precision. These beliefs are represented by the set of probability distributions $P$, where $P$ is a subset of the $N$ dimensional probability simplex $\Delta \equiv[0,1]^{N}$. A generic element of $P$ is a probability distribution $p=\left(p_{1}, p_{2}, \ldots, p_{N}\right)$, and one can think of $P$ as representing both information and confidence in that information. ${ }^{4}$ If DM has no information that allows a winnowing of the set $P$, then there is total ambiguity, characterized by $P=\Delta$. DM only knows the probability of each state is between 0 and 1 . At the other extreme, DM has

\footnotetext{
${ }^{4}$ Gajdos et al. (2004) provide a complementary interpretation of the set of probabilities. The decision-maker in their model maximizes the minimum expected utility computed with respect to a subset of the set of initially given priors. The extent to which the set of initially given priors is reduced is a measure of aversion to information imprecision.
} 
information and confidence in it which leads to the belief that only one distribution is valid. The set $P$ is a singleton, and DM faces pure risk.

DM has alpha maxi-min expected utility preferences, ${ }^{5}$ abbreviated as $\alpha$-MMEU. This implies the utility $V(x)$ from action $x$ is given by

$$
V(x)=\alpha \min _{p \in P}\left\{\sum_{\theta \in \Theta} p_{\theta} u_{\theta}^{x}\right\}+(1-\alpha) \max _{p \in P}\left\{\sum_{\theta \in \Theta} p_{\theta} u_{\theta}^{x}\right\} .
$$

When $\alpha$-MMEUis applied, the expected utility associated with a given action $x$ is calculated for each admissible probability distribution $p \in P$, with all resulting calculations being discarded except for the minimum and maximum. The parameter $\alpha=\left[1_{-}^{-}\right.$characterizes DM's degree of pessimism, as in the Arrow-Hurwicz criterion. This parameter determines the weight given to the minimum and maximum expected utilities provided by action $x$. Finding utility $V(x)$ for each action $x$ in this manner, DM selects action $x$ that maximizes $V(x)$.

When $\alpha=1, \alpha$-MMEU preferences have the MMEU form. DM is totally pessimistic. One can say DM exhibits total ambiguity aversion in this case because the existence of multiple potentially applicable probability distributions is translated into the presumption that the least favorable distribution applies. In contrast, $\alpha=0$ implies DM is totally ambiguity tolerant, presuming the most optimistic probability distribution applies. As $\alpha$ increases from zero to one, $\mathrm{DM}$ changes from being ambiguity tolerant to being ambiguity intolerant. Although $\alpha-\mathrm{MMEU}$ is more general than the MMEU model, it shares some of its shortcomings. Specifically, $\alpha-$ MMEU still ignores almost all of the information contained in the DM's set of priors; preferences are completely characterized by the best and worst scenarios but nothing between these two possibilities.

\footnotetext{
${ }^{5}$ Ghirardato, Maccheroni and Marinacci (2004) provide an axiomatic characterization of $\alpha$ - MMEU preferences.
} 
Imposing the requirement that $P$ is the core of a simple capacity allows a simple parameterization of the degree of ambiguity and, as will become clear below, places our model on a solid axiomatic ground. (See Appendix A for definitions of a capacity, a core of a capacity, and a supermodular capacity.) Given $f \equiv(f(\{1\}), \ldots, f(\{N\})) \in \Delta$ and $\lambda \in \boldsymbol{\phi}, 1_{-}^{-}$, a capacity $v$ is

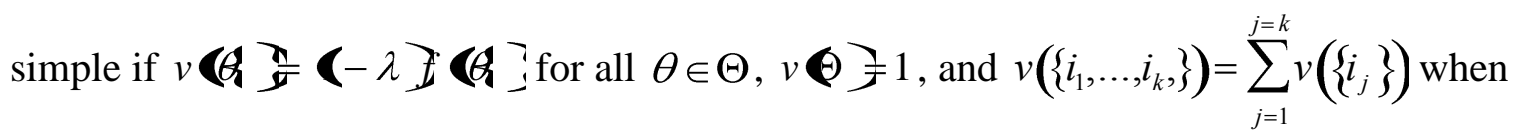
$k<N$. The probability distribution $f$ is called an anchor. A simple capacity is supermodular and $P=\{(1-\lambda) f\}+\lambda \Delta$. That is, $P$ is the Minkowski sum of a singleton set $(-\lambda \rrbracket$ and the probability simplex $\Delta$ scaled by $\lambda$. These assumptions imply $P$ is a simplex with faces parallel to those of simplex $\Delta$, and the variable $\lambda$ is naturally interpreted as a measure of the degree of ambiguity. In Bayesian statistics set $P$ is called an $\varepsilon$-contaminated set of priors (Berger and Berliner, 1986).

Simple capacities constitute a special case of neo-additive capacities (Chateauneuf, Eichberger and Grant, 2007). Recently, Chateauneuf, Eichberger and Grant (2007) have axiomatized CEU preferences with neo-additive capacity. It turns out that DM has CEU preferences with simple capacity $v$ if and only if DM has $\alpha$-MMEU with beliefs given by the core of capacity $v$ (Ludwig and Zimper, 2006, Chateauneuf, Eichberger and Grant, 2007). Thus, the preferences employed in this paper stem from the behavioral axioms employed in Chateauneuf, Eichberger and Grant (2007). Under these axioms the coefficient of pessimism $\alpha$ is constant over the space of all acts. This is in contrast to Ghirardato, Maccheroni, and Marinacci's (2004) axiomatization of $\alpha$-MMEU preferences where if one takes the set of probability distributions derived from their partial order of independent acts as DM's beliefs then coefficient $\alpha$ in general depends on the act over which the $\alpha-\mathrm{MMEU}$ preference functional is evaluated (Eichberger, Grant and Kelsey, 2008). Since having a constant $\alpha$ considerably simplifies our comparative static analysis we have adopted belief structure represented by a simple capacity. Moreover, as stated in the following theorem (for a proof, see Ludwig and Zimper, 2006) the $\alpha$-MMEU preference functional with respect to the core of a simple capacity has a very compact and easy-to-analyze form. 
Theorem 1 (Parameterizing Ambiguity): Let $\theta_{i}$ be defined by $\theta_{i} \in \mathcal{1}_{\mathbf{A}} \ldots, N, \theta_{i} \neq \theta_{j}$ for $i \neq j$ with $i, j \in 1_{3} \ldots, N^{-}$, and $u_{\theta_{1}}^{x} \leq u_{\theta_{2}}^{x} \leq \ldots \leq u_{\theta_{N-1}}^{x} \leq u_{\theta_{N}}^{x}{ }^{6}$ If $P$ is the core of simple capacity $v$, then

$$
V(x)=(1-\lambda)\left\{f\left(\left\{\theta_{1}\right\}\right) u_{\theta_{1}}^{x}+f\left(\left\{\theta_{2}\right\}\right) u_{\theta_{2}}^{x}+\ldots .+f\left(\left\{\theta_{N}\right\}\right) u_{\theta_{N}}^{x}\right\}+\lambda\left\{u_{\theta_{N}}^{x}-\alpha\left(u_{\theta_{N}}^{x}-u_{\theta_{1}}^{x}\right)\right\}
$$

Our parameter $\lambda$ corresponds to Chateauneuf, Eichberger and Grant's (2007) parameter $\delta$ while our parameter $\alpha$ corresponds to their $(1-\alpha)$. Theorem 1 indicates that, when the set of beliefs $P$ is restricted to the core of a simple capacity, the $\alpha$ - MMEU utility level for an action is a convex combination of the utility obtained from applying the anchor probability distribution and the utility obtained when all probability distributions are admissible. Because the variable $\lambda$ is the weight given to these two utility extremes, theorem 1 indicates it is a measure of the degree of ambiguity when preferences are $\alpha$ - MMEU.

To illustrate the applicability of Theorem 1 to our model of religious choice, consider three special cases: (1) $\lambda=0$; (2) $\lambda=1, \alpha=1$; and (3) $\lambda=1, \alpha=0$. For case 1 , there is no ambiguity, the anchor probability distribution applies, the degree of pessimism does not affect the evaluation of the alternatives, and the decision criterion reduces to subjective expected utility. Let the anchor probability distribution be $f=(0.8,0.1,0.1)$ for religions $X=(a, b, c)$ presented in Table 1. Then, utility representation (2) implies $V(a)=4, V(b)=-7$, and $V(c)=-14$, and religion $a$ is chosen. For case 2, ambiguity is complete and DM resolves uncertainty using max-min decision rule because she is a total pessimist. The utility function (2) implies $V(a)=-20, V(b)=-10$, and $V(c)=-20$, and religion $b$ is chosen. For case 3, ambiguity is complete and DM resolves uncertainty using max-max decision rule because she is

\footnotetext{
${ }^{6}$ Note that we slightly abuse our notation by occasionally subsuming the dependence of $\theta$ on the choice of action $x$.
} 
a total optimist. The utility function (2) implies $V(a)=10, V(b)=20$, and $V(c)=30$, and religion $c$ is chosen. This illustrates how changes in the degree of ambiguity and changes in the degree of pessimism can alter religious choice, which we now examine in more detail.

\section{Interacting Effects of the Degree of Pessimism and the Degree of Ambiguity on Utility}

Having a measure of the degree of ambiguity allows us to examine its effect on utility, and more interestingly examine how changes in the degree of ambiguity interact with changes in the degree of pessimism. The marginal effect of the degree of ambiguity on the utility provided by a particular action $x$ is given by

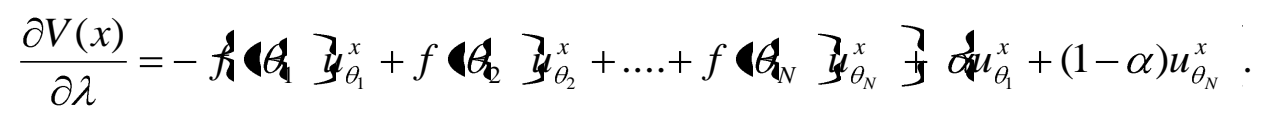

Using (3), the following result is readily obtained.

Theorem 2 (Ambiguity, Pessimism, and Utility): For any set of beliefs $P$ and any action $x \in X$, there exists a degree of pessimism $\hat{\alpha}(x)$ such that $\alpha\left(\begin{array}{l}< \\ = \\ >\end{array}\right) \hat{\alpha}(x) \Leftrightarrow \frac{\partial V(x)}{\partial \lambda}\left(\begin{array}{l}\geq \\ = \\ \leq\end{array}\right) 0$, and $\alpha\left(\begin{array}{l}< \\ >\end{array}\right) \hat{\alpha}(x) \Leftrightarrow \frac{\partial V(x)}{\partial \lambda}\left(\begin{array}{l}> \\ =\end{array}\right) 0$ when $u_{\theta_{N}}^{x}>u_{\theta_{1}}^{x}$

Proof of Theorem 2: Since $u_{\theta_{N}}^{x}-u_{\theta_{1}}^{x} \geq 0$ the derivative $\frac{\partial V(x)}{\partial \lambda}$ in (3) is non-increasing in $\alpha$.

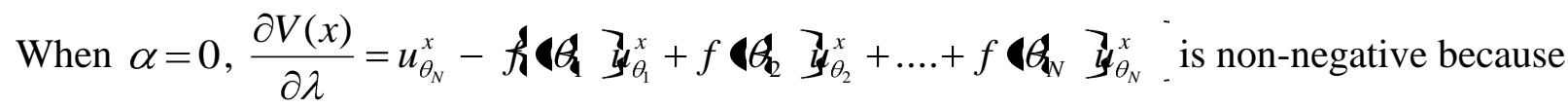
$u_{\theta_{1}}^{x} \leq u_{\theta_{2}}^{x} \leq \ldots \leq u_{\theta_{N-1}}^{x} \leq u_{\theta_{N}}^{x}$, and $\frac{\partial V(x)}{\partial \lambda}$ is strictly positive when $u_{\theta_{N}}^{x}>u_{\theta_{1}}^{x}$. Analogously, when

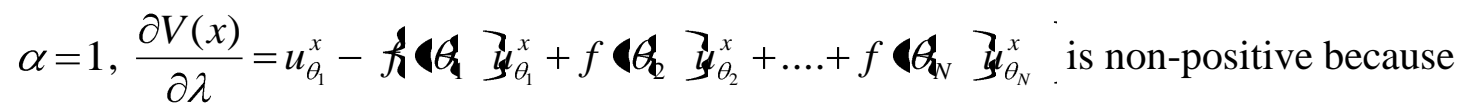
$u_{\theta_{1}}^{x} \leq u_{\theta_{2}}^{x} \leq \ldots \leq u_{\theta_{N-1}}^{x} \leq u_{\theta_{N}}^{x}$, and $\frac{\partial V(x)}{\partial \lambda}$ is strictly negative when $u_{\theta_{N}}^{x}>u_{\theta_{1}}^{x}$. Because $\frac{\partial V(x)}{\partial \lambda}$ is a 
continuous and strictly increasing function of $\alpha$, there must exist a value $\hat{\alpha}(x)$ such that $\alpha\left(\begin{array}{l}< \\ >\end{array}\right) \hat{\alpha}(x) \Leftrightarrow \frac{\partial V(x)}{\partial \lambda}\left(\begin{array}{l}\geq \\ = \\ \leq\end{array}\right) 0$, and $\alpha\left(\begin{array}{l}< \\ = \\ >\end{array}\right) \hat{\alpha}(x) \Leftrightarrow \frac{\partial V(x)}{\partial \lambda}\left(\begin{array}{l}> \\ >\end{array}\right) 0$ when $u_{\theta_{N}}^{x}>u_{\theta_{1}}^{x}$. QED

For the remainder of the discussion in this section, assume there is some variation in the payoffs associated with the action $x$ so that $u_{\theta_{N}}^{x}>u_{\theta_{1}}^{x}$ and the strict inequality of the theorem holds. In this generic case, Theorem 2 indicates that the qualitative impact of a change in the degree of ambiguity depends upon the degree of pessimism. When DM is sufficiently pessimistic, the utility of any action decreases as ambiguity increases. Conversely, when DM is sufficiently optimistic, the utility of any action increases as the degree of ambiguity increases. At the threshold degree of pessimism $\hat{\alpha}$ a change in the degree of ambiguity does not affect the utility associated with action $x$. This threshold is related to the payoffs and the anchor

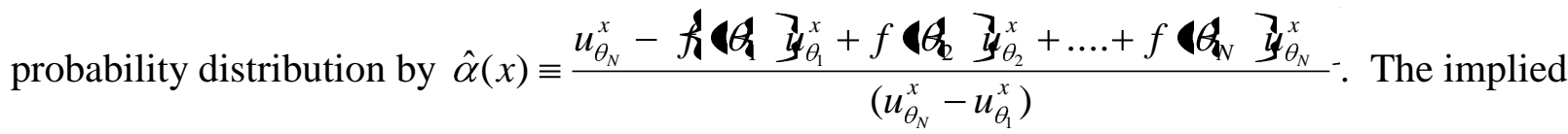
$\varangle, \lambda^{-}$isopayoff mapping is presented in Figure 1, and the calculations that prove the mapping has this structure are presented in Appendix B.

Figure 1: Isopayoff Mapping for the Degree of Pessimism $\alpha$ and Degree of Ambiguity $\lambda$

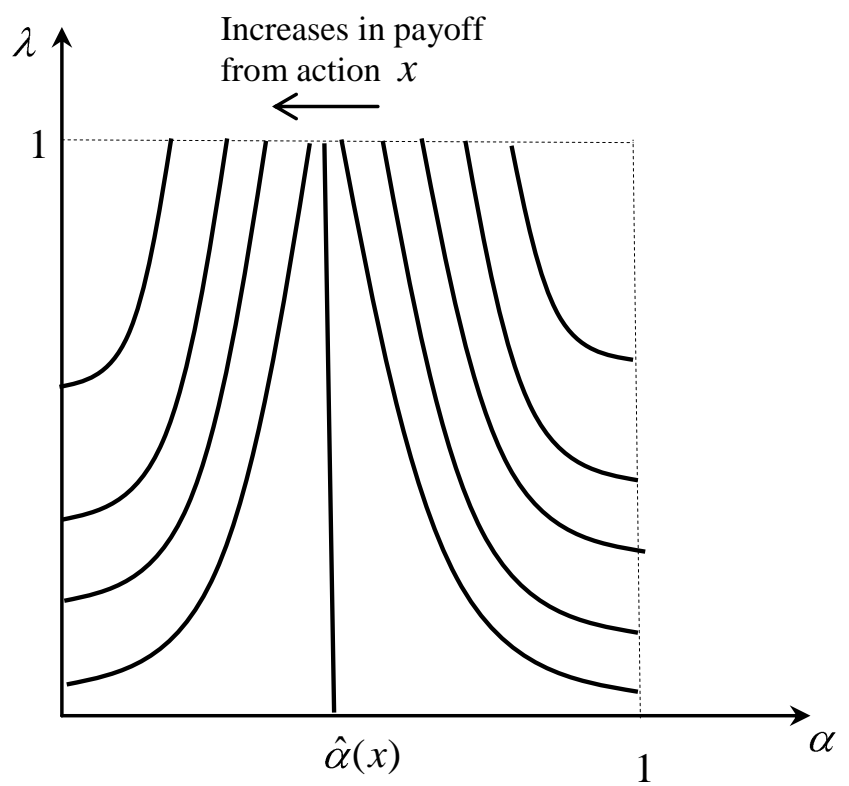


Theorem 2 is an intuitive result. An increase in ambiguity enlarges the set of admissible probability distributions $P$. Some of the added distributions will assign higher probabilities to outcomes that are more attractive and some will assign higher probabilities to outcomes that are less attractive. This implies $\max _{p \in P}\left\{\sum_{\theta \in \Theta} p_{\theta} u_{\theta}^{x}\right\}$ in the preference functional (1) will increase, while $\min _{p \in P}\left\{\sum_{\theta \in \Theta} p_{\theta} u_{\theta}^{x}\right\}$ will decrease. A more optimistic decision maker places more weight on the former, whereas the pessimistic decision maker places more weight on the latter. Thus, an increase in ambiguity will increase utility when the optimist is optimistic enough, while it will decrease utility when the pessimist is pessimistic enough.

To apply Theorem 2 to our theory of religious choice, consider religion $a$ in Table 1 . The extreme payoffs are $u_{\theta_{N}}^{a}=10$ and $u_{\theta_{1}}^{a}=-20$, and the expected utility of religion $a$ under no

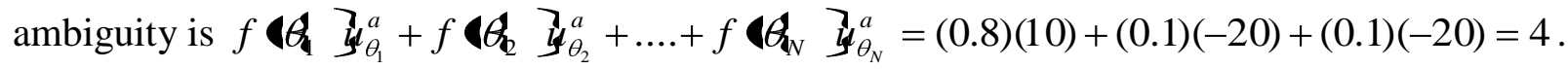
This implies $\hat{\alpha}(a)=\llbracket 0-4 \rrbracket \llbracket 0--20 \exists 0.20$. For religions $b$ and $c, \hat{\alpha}(b)=0.90$ and $\hat{\alpha}(c)=0.88$. Thus, Theorem 2 indicates DM must be quite optimistic $(\alpha<0.20)$ in order for an increase in ambiguity to increase the utility associated with religion $a$, while DM can be rather pessimistic (e.g., $\alpha=0.87$ ) and an increase in ambiguity will increase the utilities associated with religions $b$ and $c$. With a moderate degree of pessimism (e.g., $0.21<\alpha<0.87$ ), an increase in ambiguity will decrease the utility associated with religion $a$ while increasing the utilities associated with religions $b$ and $c$. This illustrates the finding that, because the marginal impact of a change in the degree of ambiguity varies across the religions, a change in ambiguity can change DM's religious choice. 


\section{The Anchor Choice, Max-min Choice, and Max-max Choice}

To make our notation more compact we re-write (2) in the form

$$
V(x ; \lambda, \alpha)=(1-\lambda) A(x)+\lambda B(x, \alpha),
$$

where $A(x) \equiv f\left(\left\{\theta_{1}(x)\right\}\right) \boldsymbol{u}_{\theta_{1}(x)}^{x}+f\left(\left\{\theta_{2}(x)\right\}\right) \boldsymbol{u}_{\theta_{2}(x)}^{x}+\ldots .+f\left(\left\{\theta_{N}(x)\right\}\right) \boldsymbol{u}_{\theta_{N}(x)}^{x}$ and $B(x, \alpha) \equiv u_{\theta_{N}(x)}^{x}-\alpha\left(u_{\theta_{N}(x)}^{x}-u_{\theta_{1}(x)}^{x}\right)$. Now, fix the degree of pessimism at an arbitrary level $\alpha \in[0,1]$ and consider two actions $x$ and $y$. If $A(x) \geq A(y)$ and $B(x, \alpha) \geq B(y, \alpha)$, then action $x$ is preferred to $y$ irrespective of the degree of ambiguity. If $A(x)>A(y)$ and $B(x, \alpha)<B(y, \alpha)$, then action $x$ is preferred to $y$ if and only if DM has sufficiently unambiguous beliefs. In general, DM will choose the action for which $A$ is highest when beliefs are sufficiently unambiguous, while the action for which $B$ is highest will be chosen when beliefs are sufficiently ambiguous. When the degree of ambiguity is high, the degree of pessimism points DM either toward the optimistic choice $\underset{x}{\operatorname{argmax}}\left\{u_{\theta_{N}(x)}^{x}\right\}$ or toward the pessimistic choice $\operatorname{argmax}\left\{u_{\theta_{1}(x)}^{x}\right\}$. These observations are summarized more carefully in the following theorem.

Theorem 3: (Anchor, max-max, max-min) Given any set of beliefs $P$,

(i.) For any degree of pessimism $\alpha \in[0,1]$, there exists a degree of ambiguity $\lambda$, such that DM prefers action $\operatorname{argmax} A(x)$ whenever $\lambda<\underline{\lambda}$;

(ii.) For any degree of pessimism $\alpha \in[0,1]$, there exists a degree of ambiguity $\bar{\lambda}(\alpha)$ such that DM prefers action $\operatorname{argmax} B(x, \alpha)$ whenever $\lambda>\bar{\lambda}(\alpha)$;

(iii.) There exist $\alpha_{L}$ and $\lambda_{L}$ such that DM prefers action $\underset{x}{\operatorname{argmax}}\left\{u_{\theta_{N}(x)}^{x}\right\}$ whenever $\alpha \leq \alpha_{L}$ and $\lambda>\lambda_{L}$

(iv.) There exist $\alpha_{H}$ and $\lambda_{H}$ such that DM prefers action $\underset{x}{\operatorname{argmax}}\left\{u_{\theta_{1}(x)}^{x}\right\}$ whenever $\alpha \geq \alpha_{H}$ and $\lambda>\lambda_{H}$.

Proof of Theorem 3: Part (i) follows from the continuity of the payoff function $V(x ; \lambda, \alpha)$ in $\lambda$ for each action $x$ and the fact that $\underset{x}{\operatorname{argmax}} V(x ; \lambda=0, \alpha)=\underset{x}{\operatorname{argmax}} A(x)$. Part (ii) follows from the continuity of the payoff function $V(x ; \lambda, \alpha)$ in $\lambda$ for each action $x$ and the fact that 
$\operatorname{argmax} V(x ; \lambda=1, \alpha)=\operatorname{argmax} B(x, \alpha)$. Part (iii) follows from the continuity of the payoff function $V(x ; \lambda, \alpha)$ in $(\lambda, \alpha)$ for each action $x$ and the fact that $\underset{x}{\operatorname{argmax}} V(x ; \lambda=1, \alpha=0)=\underset{x}{\operatorname{argmax}}\left\{u_{\theta_{N}(x)}^{x}\right\}$. Part (iv) follows from the continuity of the payoff function $V(x ; \lambda, \alpha)$ in $(\lambda, \alpha)$ for each action $x$ and the fact that $\underset{x}{\operatorname{argmax}} V(x ; \lambda=1, \alpha=1)=\underset{x}{\operatorname{argmax}}\left\{u_{\theta_{1}(x)}^{x}\right\}$. QED

Figure 2 illustrates the implications of Theorem 3. When there is total ambiguity $(\lambda=1)$, there is a threshold level of pessimism that will lead DM to choose the pessimistic max-min $\underset{x}{\operatorname{action}} \underset{\theta_{1}(x)}{\operatorname{argmax}}\left\{u_{\theta^{x}}\right\}$, and this choice is also best if DM becomes increasingly pessimistic. When the decision maker is entirely pessimistic, the max-min action is best under total ambiguity and under less than total ambiguity down to some threshold level $\lambda_{L}$. By analogous reasoning, there are thresholds $\alpha_{H}$ and $\lambda_{H}$ that are associated with DM choosing the optimistic max-max action $\underset{x}{\operatorname{argmax}} \boldsymbol{t}_{\boldsymbol{B}_{N}}^{\mathrm{x}}$. The tradeoffs between the level of pessimism and level of ambiguity derived in Theorem 2 indicate there are sets of $(\lambda, \alpha)$ combinations that support the max-min and max-max choices, as shown in Figure 2. Finally, as the level of ambiguity decreases to zero, Theorem 3 indicates there must be a set of $(\lambda, \alpha)$ combinations that support the anchor choice $\operatorname{argmax} A(x)$, as shown in Figure 2. Figure 2 demonstrates the case where three actions are available to the decision maker, one of these actions is the anchor choice, the second action is the max-min choice, while the third action is the max-max choice. The boundaries of the three regions in Figure 2 will vary with the parameters of the model. 


\section{Figure 2: Three Special Alternative Choices}

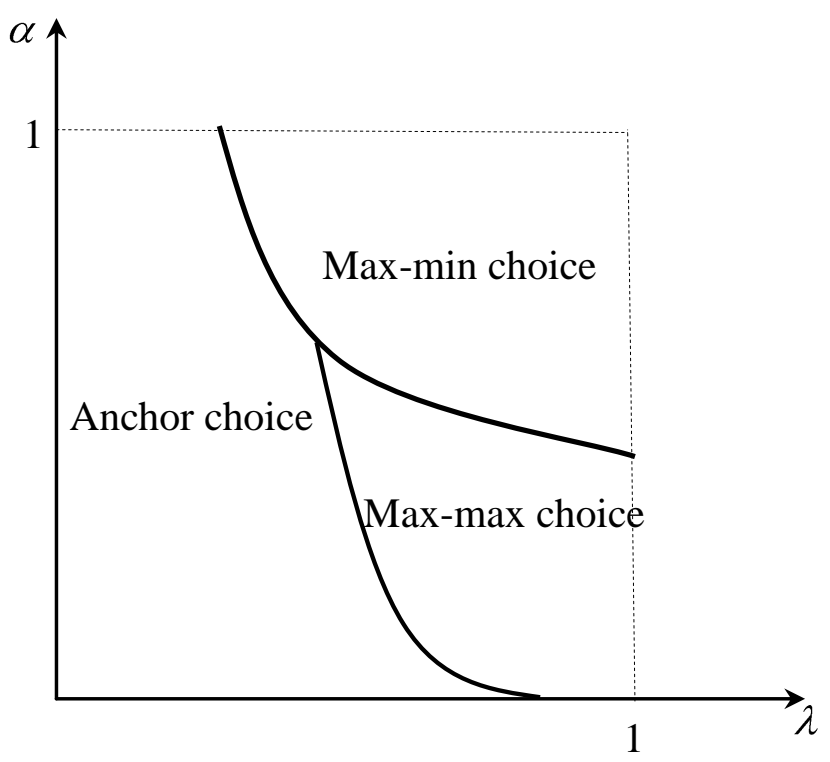

To illustrate Theorem 3 for religious choice, reconsider the religious payoff parameter values in Table 1 . When $\lambda=0, V(a ; 0, \alpha)=A(a)=4, V(b ; 0, \alpha)=A(b)=-7$, and $V(c ; 0, \alpha)=A(c)=-14$. The optimal choice is the anchor choice, religion $a$. When there is no ambiguity, the degree of pessimism does not affect the religious choice. However, consider now a low degree of pessimism $(\alpha=.1)$ and increase the degree of ambiguity. When we hit the ambiguity level $\lambda=.51$, we find $V(a ; .51, .1)=5.53, V(b ; .51, .1)=5.24$, and $V(c ; .51, .1)=5.89$, so the optimal choice switches from religion $a$ to the max-max choice, religion $c$. The optimal choice remains religion $c$ for the degree of ambiguity in the range $\lambda=60,1^{-}$. When the degree of pessimism is high $(\alpha=.9)$ and we increase the degree of ambiguity, when we hit the ambiguity level $\lambda=.53$, we find $V(a ; .53, .9)=-7.13, V(b ; .53, .9)=-7.00$, and $V(c ; .53, .9)=-14.53$, so the optimal choice switches from religion $a$ to the max-min choice, religion $b$. The optimal choice remains religion $b$ for the degree of ambiguity in the range $\lambda=\left(52,1_{-}^{-}\right.$ 
Under complete ambiguity, the preference functional (4) becomes $V(x ; 1, \alpha)=B(x, \alpha)$.

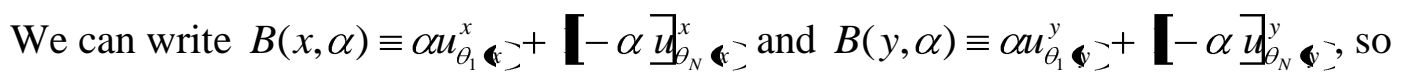
$\left.B(x, \alpha)-B(y, \alpha) \equiv \alpha \coprod_{\theta_{1}}^{x}-u_{\theta_{1}}^{y}\right][-\alpha\rceil_{\theta_{N}}^{x}$ $u_{\theta_{1}}^{x}>u_{\theta_{1}}^{y}$, then $B(x, \alpha)>B(y, \alpha)$ for all $\alpha \in \mathbf{Q}, 1_{-}^{-}$. Action $x$ provides a higher payoff than action $y$ in the most optimistic state and in the most pessimistic state. Thus, action $y$ will not be chosen over action $x$ under complete ambiguity, no matter what the degree of pessimism. This observation suggests the following definition.

Definition 1 (Irrelevance under complete ambiguity): An action $y$ is "irrelevant under complete ambiguity" if there is some other action $x$ such that $u_{\theta_{N}}^{x}>u_{\theta_{N}}^{y}$ and $u_{\theta_{1}}^{x}>u_{\theta_{1}}^{y}$;

Irrelevance is important because it reduces the number of meaningful alternatives when it applies. In particular, for religious choice, the irrelevance of many conceivable religions offers an explanation for why a given decision maker may not perceive too many relevant religions in a decision matrix. It is possible that one particular action makes all other actions irrelevant under complete ambiguity. In this special case, a move toward complete ambiguity motivates DM to select one particular choice, no matter what DM's degree of pessimism. This special case is described by the following definition.

Definition 2 (Dominance under complete ambiguity): An action $x$ is "dominant under complete ambiguity" if $u_{\theta_{N}}^{x}>u_{\theta_{N}}^{y}$ and $u_{\theta_{1}}^{x}>u_{\theta_{1}}^{y}$ for all $y \in X, y \neq x$. 
The concepts of irrelevance and dominance under complete ambiguity have implications for evangelism. If the beliefs of decision makers are completely ambiguous, then one religion will make others irrelevant by instilling the perception that the religion offers a high reward for adoption when it is true, and high penalty of non-adoption. For example, suppose the decision matrix for DM changes from what is presented in Table 1 to what is presented in Table 2. As before, religion $c$ offers the highest reward for adoption when it is true:

$u_{\theta_{N}}^{c}=30>u_{\theta_{N}}^{b}=20>u_{\theta_{N}}^{a}=10$. However, now religion $c$ also imposes the largest penalty for non-adoption when true: $u_{\theta_{1}}^{c}=-20>u_{\theta_{1}}^{b}=-30=u_{\theta_{1}}^{c}=-30$. It follows that religion $c$ is dominant under complete ambiguity, making both religions $a$ and $b$ irrelevant.

Table 2: Dominance Under Complete Ambiguity

\begin{tabular}{|l|c|c|c|}
\hline & Religion a True & Religion b True & Religion c True \\
\hline Religion a & 10 & -20 & -30 \\
\hline Religion b & -10 & 20 & -30 \\
\hline Religion c & -10 & -20 & 30 \\
\hline
\end{tabular}

When $\lambda<1$ DM's choice is affected by the difference between the payoffs for the most preferred and the least preferred states of nature, $u_{\theta_{N}}^{x}-u_{\theta_{1}}^{x}$. This is because the marginal effect of the degree of pessimism on the attractiveness of action $x$ is $-\lambda \mathbf{Y}_{\theta_{N}}^{x}-u_{\theta_{1}}^{x} \Varangle 0$, obtained by differentiating (2) with respect to $\alpha$. Greater pessimism reduces the anticipated payoff associated with any action $x$, as would be expected. More interestingly, we see this marginal impact depends upon both the degree of ambiguity $\lambda$ and the payoff difference $u_{\theta_{N}}^{x}-u_{\theta_{1}}^{x}$. For a given level of ambiguity $\lambda$, greater pessimism reduces utility more when the payoff difference 
$u_{\theta_{N}}^{x}-u_{\theta_{1}}^{x}$ is larger. And, for a given payoff difference $u_{\theta_{N}}^{x}-u_{\theta_{1}}^{x}$, greater pessimism reduces utility more when the degree of ambiguity $\lambda$ is greater. This reasoning underlies the following result.

Theorem 4 (Smallest and Largest Differential Payoffs and the Degree of Pessimism): If $u_{\theta_{N}(x)}^{x}-u_{\theta_{1}(x)}^{x} \leq u_{\theta_{N}\left(x^{\prime}\right)}^{x^{\prime}}-u_{\theta_{1}\left(x^{\prime}\right)}^{x^{\prime}}$ for all actions $x^{\prime}$ that are alternatives to action $x$, and DM prefers action $x$ to all alternative actions $x^{\prime}$ with degree of pessimism $\bar{\alpha}$ and beliefs $P$, then DM must prefer action $x$ to all alternative actions $x^{\prime}$ with higher degree of pessimism $\alpha>\bar{\alpha}$ and beliefs $P$. Conversely, if $u_{\theta_{N}(x)}^{x}-u_{\theta_{1}(x)}^{x} \geq u_{\theta_{N}\left(x^{\prime}\right)}^{x^{\prime}}-u_{\theta_{1}\left(x^{\prime}\right)}^{x^{\prime}}$ for all actions $x^{\prime}$ that are alternatives to action $x$, and DM prefers action $x$ to all alternative actions $x^{\prime}$ with degree of pessimism $\bar{\alpha}$ and beliefs $P$, then DM must prefer action $x$ to all alternative actions $x^{\prime}$ with degree of pessimism $\alpha<\bar{\alpha}$ and beliefs $P$.

Proof of Theorem 4: We need only prove the first part, for the proof for the second part is analogous. Assume $\left(u_{\theta_{N}}^{x}--u_{\theta_{1}}^{x}\right) \leq\left(u_{\theta_{N}}^{x^{\prime}},-u_{\theta_{1}}^{x^{\prime}}\right)$ for all $x^{\prime}$. Applying Theorem 1, we can write

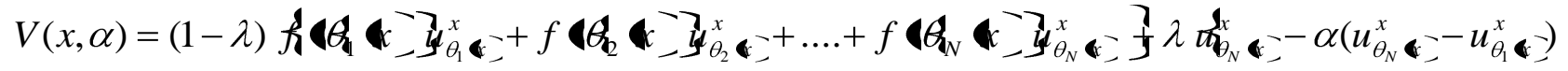
and

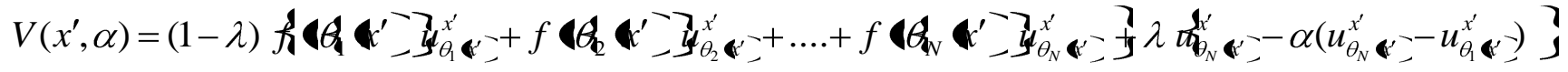
Let $W\left(x, x^{\prime}, \alpha\right) \equiv V(x, \alpha)-V\left(x^{\prime}, \alpha\right)$. By our supposition,

(i) $W\left(x, x^{\prime}, \bar{\alpha}\right) \geq 0$ for all $x^{\prime}$.

Furthermore, the assumption $\left(u_{\theta_{N}}^{x},-u_{\theta_{1}}^{x}\right) \leq\left(u_{\theta_{N}}^{x^{\prime}},-u_{\theta_{1}}^{x^{\prime}}\right)$ for all $x^{\prime}$ implies

$$
\left.\frac{\partial W\left(x, x^{\prime}, \alpha\right)}{\partial \alpha}=-\lambda \boldsymbol{u}_{\theta_{N}}^{x}--u_{\theta_{1}}^{x}\right)-\left(u_{\theta_{N}}^{x^{\prime}}-u_{\theta_{1}}^{x^{\prime}}\right) \neq 0 \text { for all } \alpha \text { and all } x^{\prime} .
$$

It follows from (i) and (ii) that $W\left(x, x^{\prime}, \alpha\right) \geq 0$ for all $x^{\prime}$ and all $\alpha$ such that $\bar{\alpha} \leq \alpha \leq 1$. That is, any decision maker with degree of pessimism $\alpha>\bar{\alpha}$ must also prefer religion $x$. QED.

Theorem 4 indicates that optimists are more likely than pessimists to find appeal in a choice with a large difference between the largest possible payoff and the lowest. If such a 
choice is appealing, it will tend to capture the most optimistic decision makers. Conversely, pessimists will tend to find more appeal in a choice where this difference is small. If a choice with such a small difference in the possible payoffs is attractive, Theorem 4 indicates it will be the most pessimistic decision makers who will find it attractive.

To illustrate Theorem 4 for religious choice, when the level of the ambiguity for the decision maker with the Table 1 payoff structure is $\lambda=.50$, we find $V(a ; .50, .11)=5.35$, $V(b ; .50, .11)=4.85$, and $V(c ; .50, .11)=5.25$, so the optimal choice is religion $a$. Religion $a$ has the smallest payoff difference, with $\left(u_{\theta_{N}}^{a},-u_{\theta_{1}}^{a}\right)=10--20=30$. Theorem 4 indicates that religion $a$ would be the optimal choice for any higher degree of pessimism $\alpha \in(.11,1]$, which trial calculations can confirm. When the level of the ambiguity is $\lambda=.51$, we find $V(a ; .51, .1)=5.53, V(b ; .51, .1)=5.24$, and $V(c ; .51, .1)=5.89$, so the optimal choice is religion $c$.

Religion $c$ has the largest payoff difference, with $\left(u_{\theta_{N}}^{c}-u_{\theta_{1}}^{c}\right)=30-420=50$. Theorem 4 indicates that religion $c$ would be the optimal choice for any lower degree of pessimism $\alpha \in[0, .10)$, which trial calculations can confirm.

\section{Discussion}

John von Neumann and Blaise Pascal lived in different centuries, but are connected in a number of ways. Both were great mathematicians, Pascal in $17^{\text {th }}$ century, von Neumann in the $20^{\text {th }}$. Both made significant contributions to decision theory, Pascal with his wager and work on probability, von Neumann with his minimax theorem of 1928 and his Theory of Games and Economic Behavior with Oscar Morgenstern (Morgenstern and von Neumann, 1944[2000]). Both died relatively young, Pascal at 39, von Neuman at 54. A final interesting connection is Pascal's application of decision theory to religion may have influenced the religious choice of 
von Neuman. In the summer of 1955 , von Neumann was diagnosed with advanced, incurable cancer. By the time the disease had confined him to bed, von Neumann had converted to Catholicism. About this conversion, Jorden (1994a, p.1) comments, "As might be expected of the inventor of the minimax principle, von Neumann was reported to have said, perhaps in part jovially, that Pascal had a point: If there is a chance that God exists, and that damnation is the lot of the unbeliever, then it is reasonable to believe."

Applying our version of the $\alpha$-MMEUmodel, a model in the decision theoretic tradition of Pascal and von Neumann, we have developed a relatively general theory of religious choice. In doing so, we have addressed the concern of Iannaccone $(1998,1491)$ who notes that "the problem of religious uncertainty has received little attention and scarcely any formal analysis." The theory of religious choice embedded in our model is one that recognizes uncertainty in the form of ambiguity, not just risk. This is significant in that, in the extreme case of pure risk (i.e., no ambiguity), the decision maker's degree of pessimism has no impact on choice. In the general case, the degree of ambiguity interacts with the decision maker's degree of pessimism, so a change in one may or may not alter the religion chosen, depending upon the level of the other.

A recent Wikipedia entry reports that Christianity and Islam are the two largest religions in the world, with 2.1 billion and 1.5 billion followers, respectively (Wikipedia, 2008). By comparison, the number of Atheists is small, included among the 1.1 billion classified in the catch all category atheist/anti-theistic/anti-religious/secular/agnostic. How might our theory offer an explanation for these numbers?

Suppose the typical decision maker perceives Atheism, Christianity, and Islam as religious alternatives. Simplistically speaking, Christianity and Islam each offer a heavenly 
afterlife for adopting the true religion, and each offer a hellish afterlife for not adopting the true religion. Assume this leads the decision maker to associate the lowest maximum payoff and lowest minimum payoff with Atheism. Under complete ambiguity, this implies Atheism will neither be the max-max choice of the extreme optimist, nor the max-min choice of the extreme pessimist. Thus, significant ambiguity is one possible explanation for the small number of Atheists compared to the number of adherents to Christianity and Islam.

The utilized model indicates that the saliency of religious reward concepts like heaven and religious punishment concepts like hell tends to be augmented by increased ambiguity. Under complete ambiguity, the perception of heaven for correct adoption attracts optimists, while the perception of hell for incorrect non-adoption attracts pessimists. Thus, a move toward ambiguity tends to prompt a decision maker away from Atheism, either toward Christianity or Islam. To adopt Atheism, a move away from complete ambiguity must be made, but such a move is not sufficient. The decision maker must also have an anchor probability distribution that places little probability weight on the truth of either Christianity or Islam, but much weight on the truth of Atheism.

The studied parameterization of the $\alpha$-MMEUmodel can be applied to more than religious choice. The model is especially suited to situations where one might expect an interaction between the degree of ambiguity and degree of pessimism. Also, it is interesting to note that, if we think of the decision under uncertainty as that being made by an agent in a principle-agent problem, the principle may be able to control the behavior of the agent more effectively by taking action that will increase the degree of ambiguity in the agent's mind. This counter-intuitive result arises from the fact that a move toward ambiguity increases the saliency 
of the highest and lowest possible outcomes associated with a decision alternative, which the principle may also be able to control.

Lastly, we note that our theory of religious choice does not help us determine whether God actually exists, nor help us determine whether any particular religion is true. First of all, the choice may not be ours, but God may be the one doing the choosing, and we just think we have a choice. $^{7}$ Second, if we can choose independently, our theory cannot tell us whether we are choosing a God of our creation, or choosing a God that has created us. Our theory is consistent with the idea that people create God and religion, with the attractiveness of a given religion dependent upon the extent to which it recognizes how people make choices under varying degrees of ambiguity. However, it is also consistent with the existence of a God who values faith, and created people who can express it as they make religious choices while facing the ambiguity associated with death.

\footnotetext{
${ }^{7}$ The Calvinist perspective in Christian theology, for example, is that God must first choose to provide grace to you before you can choose God. This is in contrast to doctrines of "free will" that postulate free agency, so that you can independently choose God or not.
} 


\section{References}

Arrow, K.J. and L. Hurwicz, 1972. An optimality criterion for decision-making under ignorance. In Uncertainty and Expectations in Economics: Essays in Honour of G.L.S. Shackle, C. F. Carter and J. L. Ford (eds). Oxford: Basil Blackwell.

Berger, J. and L. Berliner, 1986. A Definition of Subjective Probability. Annals of American Statistics 34, 199-205.

Camerer, C.F. and M. Weber, 1992. Recent Developments in Modeling Preferences: Uncertainty and Ambiguity. Journal of Risk and Uncertainty 5(4), 325-70.

Camerer, C.F., 1999. Ambiguity-Aversion and Non-Additive Probability: Experimental Evidence, Models and Applications. In L. Luini (ed.), Uncertain Decisions: Bridging Theory and Experiments. Kluwer Academic Publishers, 51-79.

Chateauneuf, A., Eichberger, J. and S. Grant, 2007. Choice under uncertainty with the best and worst in mind: Neo-additive capacities. Journal of Economic Theory 137, 538 - 567.

Cohen, M., 1992. Security level, potential level, expected utility: A three-criteria decision model under risk. Theory and Decision 33(2), $101-134$.

Eichberger, J., Grant, S. and D. Kelsey, 2008. Differentiating ambiguity: an expository note. Forthcoming in Economic Theory.

Ellsberg, D. 1961. Risk, Ambiguity and the Savage Axioms. Quarterly Journal of Economics 75:64369.

Gajdos, T., Tallon, J.M, and Vergnaud, J.C., 2004. Decision making with imprecise probabilistic information. Journal of Mathematical Economics, 40, 647-681.

Ghirardato, P., Klibanoff, P. and M. Marinacci, 1998. Additivity with Multiple Priors. Journal of Mathematical Economics 30, 405-420.

Ghirardato, P., Maccheroni, F., and Marinacci, M., 2004. Differentiating ambiguity and ambiguity attitude. Journal of Economic Theory 118(2), 133-73.

Gilboa, I., and Schmeidler, D., 1989. Maxmin expected utility with non-unique prior. Journal of Mathematical Economics 18, 141-153.

Hacking, I., 1994. The logic of Pascal's wager, Gambling on God: Essays on Pascal's Wager. Chapter 3, Jeff Jorden (ed.), Rowen \& Littlefield: London.

Iannaccone, L. R., 1998. Introduction to the economics of religion. Journal of Economic Literature 36 (3), 1465-1497.

Jaffray, J.-Y. and F. Philippe, 1997. On the existence of subjective upper and lower probabilities. Mathematics of Operations Research 22(1), 165 - 185.

Jorden, J., 1994a. Introduction, Gambling on God: Essays on Pascal's Wager. Jeff Jorden (ed.), Rowen \& Littlefield: London. 
Jorden, J., 1994b. The many Gods objection, Gambling on God: Essays on Pascal's Wager. Chapter 8, Jeff Jorden (ed.), Rowen \& Littlefield: London.

Keynes, J.M. 1921. A Treatise on Probability. London: Macmillan.

Knight, F., 1921[1971]. Risk, Uncertainty, and Profit. University of Chicago Press: Chicago, IL.

Ludwig, A. and A. Zimper, 2006. Rational expectations and ambiguity: a comment on Abel (2002). Economics Bulletin 4 (2), 1-15.

Machina, M. J. and D. Schmeidler. 1992. A more robust definition of subjective probability. Econometrica 60 (4), 745-780.

Montgomery, J.D., 1996. Contemplations on the economic approach to religious behavior._American Economic Review 86 (2), 443-448.

Morgenstern, O. and J. von Neumann, 1944[2000]. Theory of Games and Economic Behavior,.Princeton University Press: Princeton, NJ.

Morris, T., 1994. Wagering and evidence, Gambling on God: Essays on Pascal's Wager. Chapter 5, Jeff Jorden (ed.), Rowen \& Littlefield: London.

Olszewski, W., 2007. Preferences over Sets of Lotteries. Review of Economic Studies 74, 567-595.

Pascal, B., 1670 [1958]. Pascal's Pensées. E.P. Dutton \& Co., Inc.: New York, NY.

Quinn, P.L., 1994. Moral objections to Pascalian wagering, Gambling on God: Essays on Pascal's Wager. Chapter 6, Jeff Jorden (ed.), Rowen \& Littlefield: London.

Savage, L.J., 1954. The Foundations of Statistics. Wiley: New York, NY.

Schmeidler, D., 1989. Subjective Probability and Expected Utility without Additivity. Econometrica 57, 571-587.

Siniscalchi, M., 2006. A Behavioral Characterization of Plausible Priors. Journal of Economic Theory $128,91-135$.

Smith, A. 1759[2000]. The Theory of Moral Sentiments. Prometheus Books: Amherst, NY.

Wikipedia, 2008. Encyclopedic entry for "Major Religious Groups" at http://en.wikipedia.org/wiki/Major_religious_groups 


\section{Appendix A}

Let $\Theta$ denote a finite state space $\{1, \ldots, N\}$ and let $\Sigma$ denote the $\sigma$-algebra of all subsets of $\Theta$; i.e., $\Sigma=2^{\Theta}$. Let $\Delta$ denote the set of all additive probability measures over $\Sigma$. A capacity is a nonadditive set function $v: \Sigma \rightarrow[0,1]$ such that (i) $v(\varnothing)=0$, (ii) $v(\Sigma)=1$, and (iii) $v(X) \leq v(Y)$ for all $X, Y \subseteq \Sigma$ and $X \subseteq Y$. The core of the capacity $v$ is the closed, convex, and bounded set $C(v)=\{p \in \Delta: p(X) \geq v(X), \forall X \in \Sigma\} . C(v)$ is by construction polyhedral. The capacity, $v$, is supermodular (convex) if $v(X \cup Y)+v(X \cap Y) \geq v(X)+v(Y)$ for all $X, Y \subseteq \Sigma$. When $v$ is supermodular $C(v)$ is non-empty, $v$ is balanced, and $v(X)=\min \{p(X): p \in C(v)\}$ for all $X \in \Sigma$.

\section{Appendix B: Isopayoff Curve Analysis}

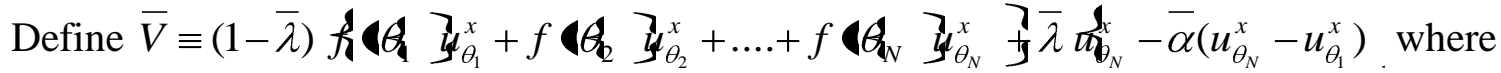
$0 \leq \bar{\alpha}, \bar{\lambda} \leq 1$, and consider the set of pairs $(\alpha, \lambda$, such that the decision-maker's payoff from action $x$ yield the same payoff, given by

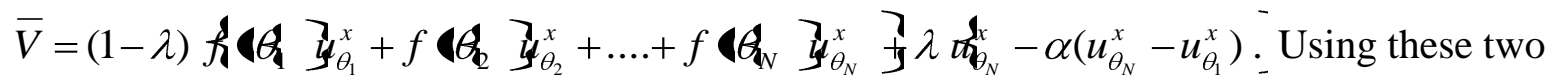
representations of $\bar{V}$ to solve for $\lambda$ in terms of $\alpha$, we obtain:

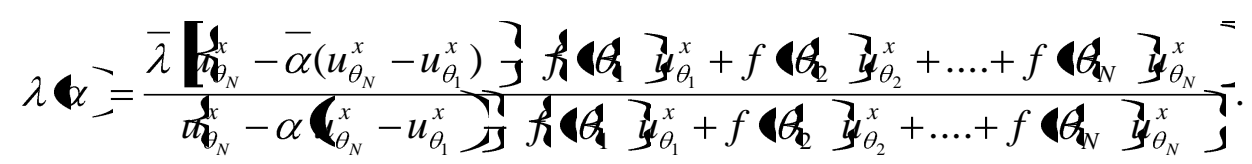

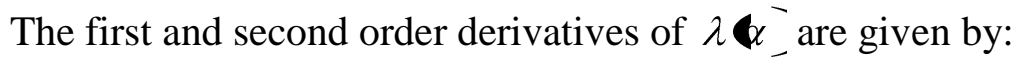

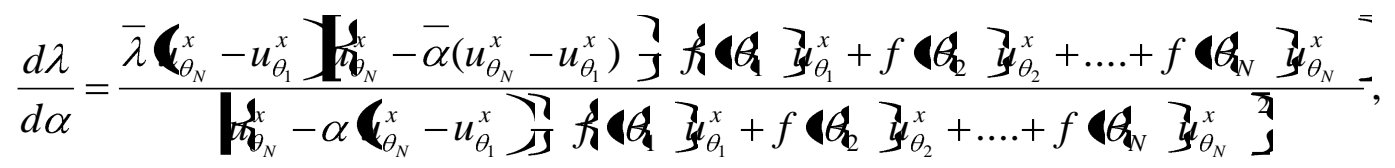

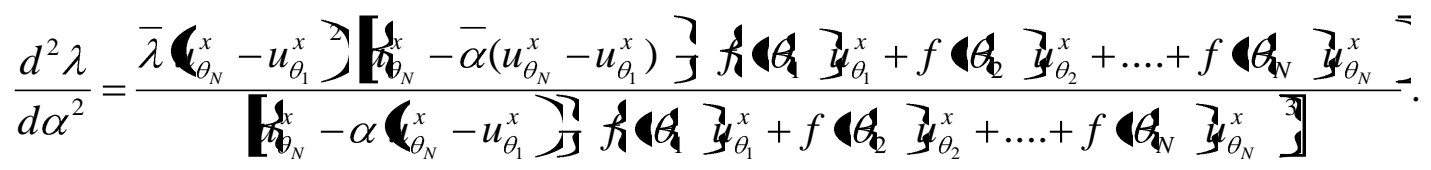




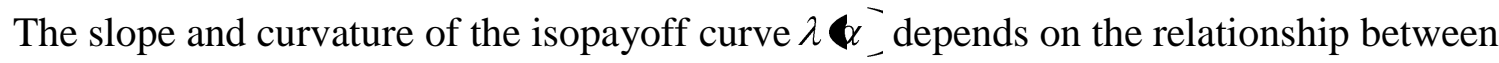

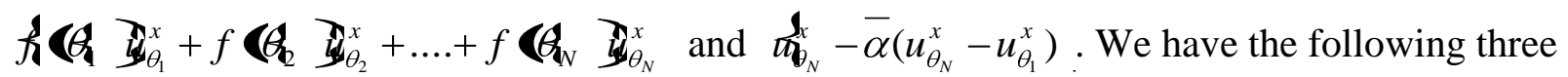
cases:

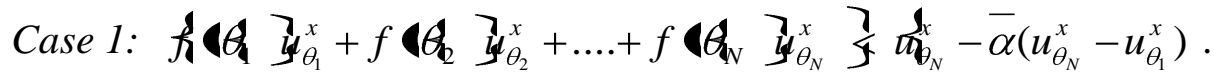

It is straightforward to verify that $\frac{d \lambda}{d \alpha}>0$ and $\frac{d^{2} \lambda}{d \alpha^{2}}>0$ over the admissible range of parameters.

The isopayoff curve for Case 1 is graphed in Figure B1.

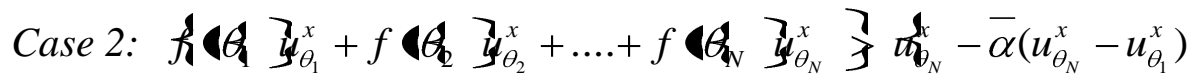
$\frac{d \lambda}{d \alpha}<0$ and $\frac{d^{2} \lambda}{d \alpha^{2}}>0$ over the admissible range of parameters (see Figure B2).

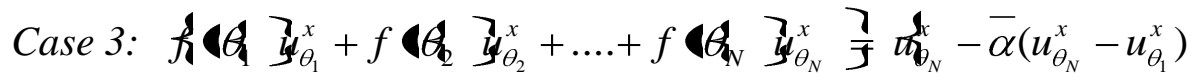

The isopayoff curve for Case 3 is the vertical line graphed in Figure B3.

We can graph the three different types of indifference curves for the three possible cases in one figure, which is done in Figure 1 in the text in the discussion after Theorem 2.

Figure B1

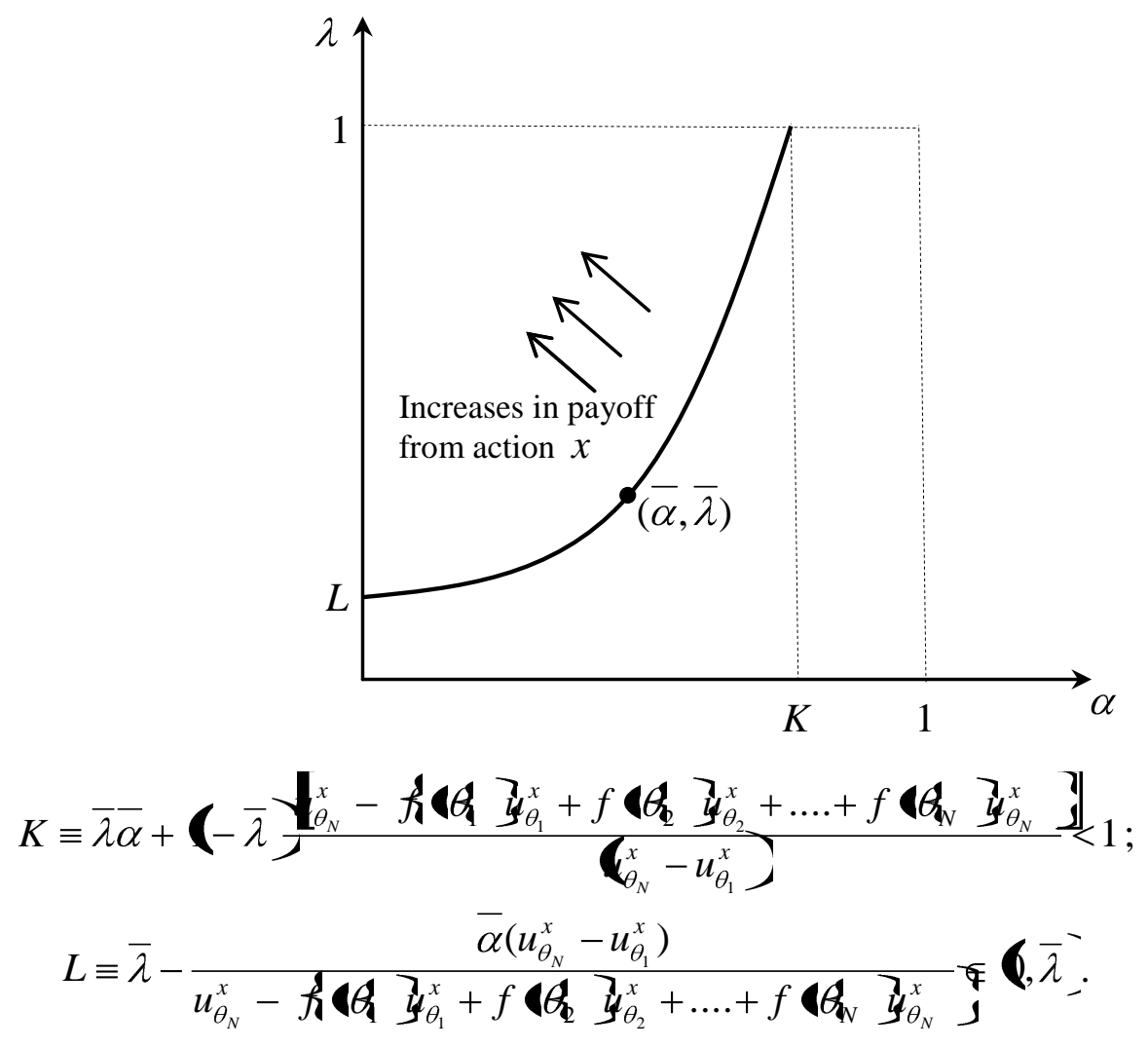


Figure B2

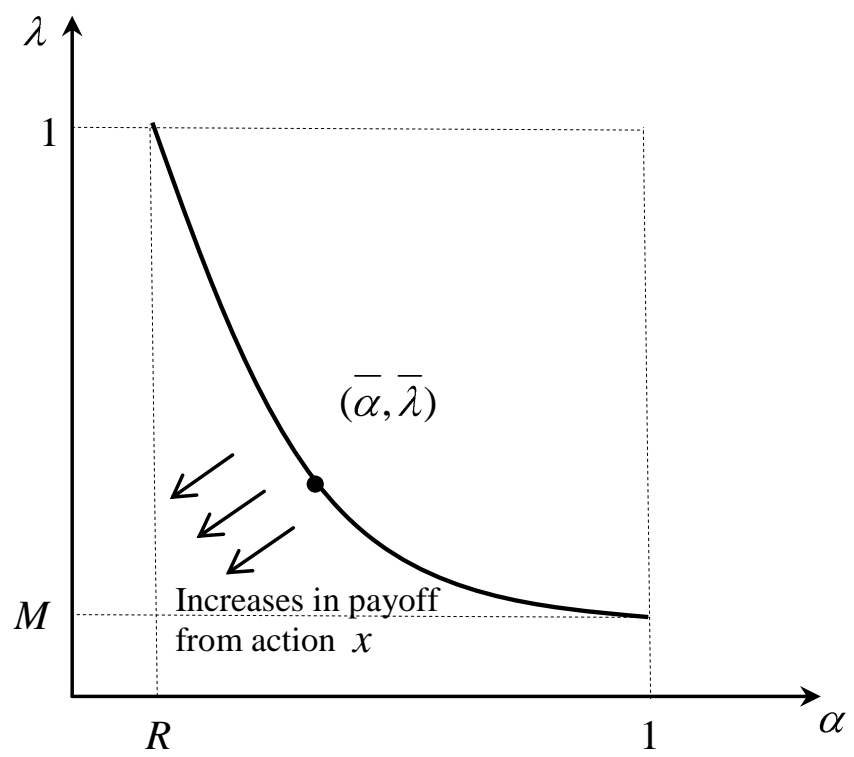

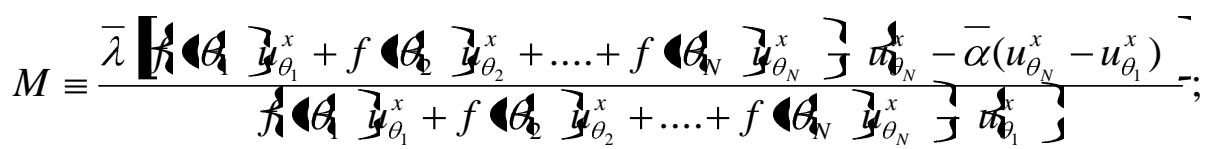

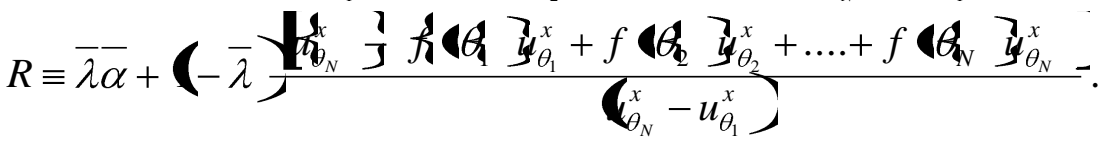

Figure B3

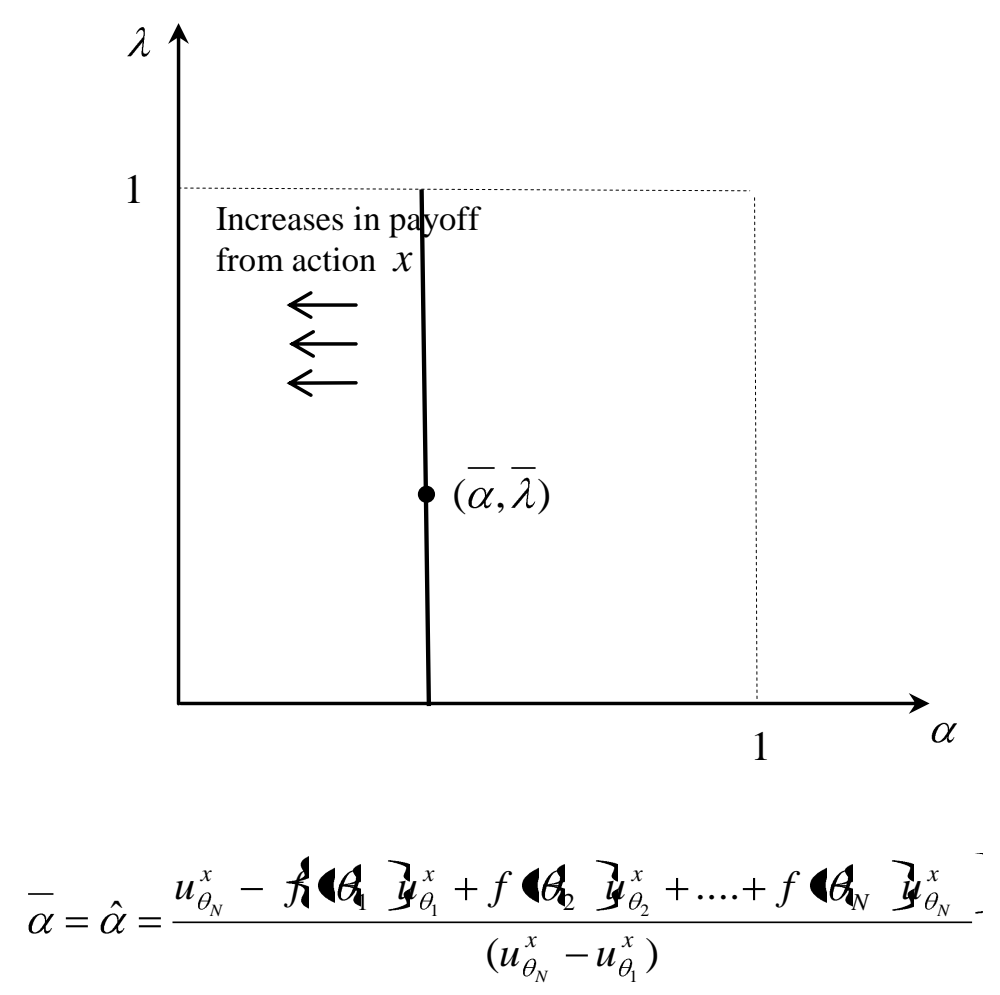




\section{Using isopayoff curves to compare actions $x$ and $x^{\prime}$ :}

Consider two actions $x$ and $x^{\prime}$ and an arbitrary $\psi, \bar{\lambda}$. Suppose that action $x$ yields a higher payoff than action $x^{\prime}$ when degree of pessimism and degree of ambiguity are given by $\left(\alpha, \bar{\lambda}^{\prime}\right.$; That is, we assume that $(1-\bar{\lambda}) A(x)+\bar{\lambda} B(x, \bar{\alpha}) \geq(1-\bar{\lambda}) A\left(x^{\prime}\right)+\bar{\lambda} B\left(x^{\prime}, \bar{\alpha}\right)$. For the case of parameters depicted n Figure B4, shaded area represents the set of $(\alpha, \lambda)$ such that $x$ yields a higher payoff than action $x^{\prime}$.

\section{Figure B4}

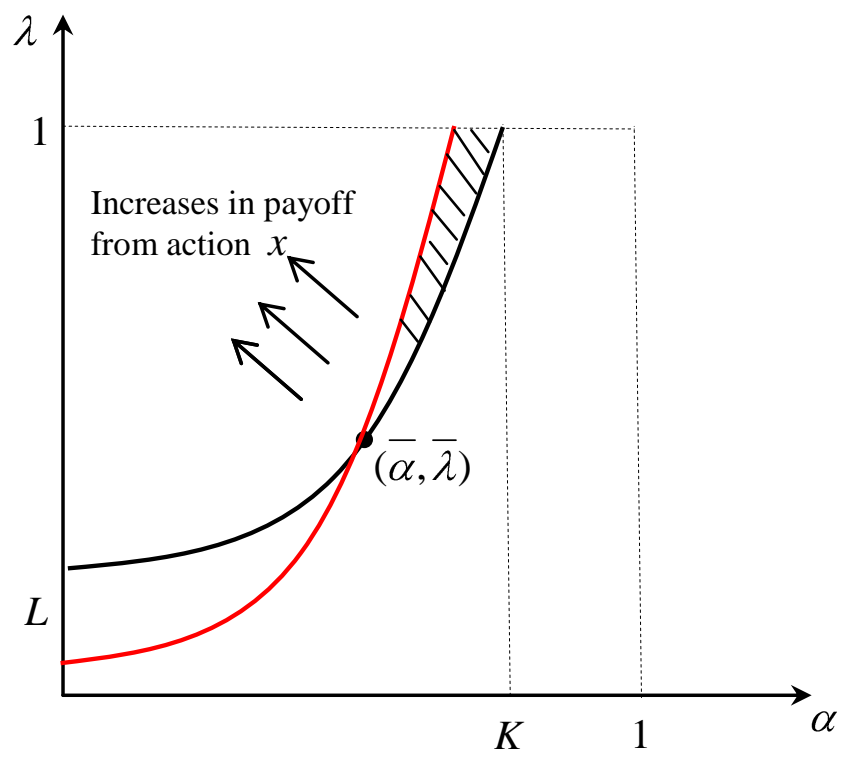

